\title{
Staggered-SAR for High-Resolution Wide-Swath Imaging
}

\author{
M. Villano*, G. Krieger*, A. Moreira* \\ *German Aerospace Center (DLR), Microwaves and Radar Institute \\ Oberpfaffenhofen, 82234 Wessling, Germany, E-mail: michelangelo.villano@dlr.de
}

Keywords: Earth observation missions, synthetic aperture radar (SAR), high-resolution wide-swath (HRWS) imaging, continuous PRI variation.

\begin{abstract}
Synthetic aperture radar (SAR) is a remote sensing technique, capable of providing high resolution images independent of weather conditions and sunlight illumination. This makes SAR very attractive for the systematic observation of dynamic processes on the Earth's surface. However, current SAR systems are limited, in that a wide coverage can only be achieved at the expense of a degraded azimuth resolution. To overcome this limitation, an innovative concept is considered, Staggered-SAR, where the pulse repetition frequency (PRF) is continuously varied. High-resolution wide-swath (HRWS) imaging is thus achieved without the need for a long antenna with multiple apertures.
\end{abstract}

\section{Introduction}

Synthetic Aperture Radar (SAR) is an established remote sensing technique, capable of acquiring high resolution images of the Earth's surface independent of weather conditions and sunlight illumination. Further unique opportunities emerge from the coherent combination of multiple SAR images. Several applications require uninterrupted time series of radar images with short time intervals between consecutive acquisitions. However, all current high-resolution SAR systems are rather limited with regard to their acquisition capability. An example is TerraSAR-X, which provides multiple imaging modes for different trade-offs between resolution and coverage (see upper part of Fig. 1): In stripmap mode (spatial resolution of 3 $\mathrm{m})$, only $2 \%$ of the Earth's landmass can be mapped during its 11 days repeat cycle. This limitation also posed a challenge in the design of the TanDEM-X mission and constrains the accuracy and resolution of the digital elevation model [5].

The lower part of Fig. 1 shows that future SAR missions may require a mapping capability one or even two orders of magnitude better than that of TerraSAR-X. A prominent example is Tandem-L, whose goal is the investigation of dynamic processes on the Earth's surface. For this, an extremely powerful SAR instrument is required, capable of continuously mapping a $350 \mathrm{~km}$ wide swath in full polarisation and with a spatial resolution well below $10 \mathrm{~m}$ [8]. Other missions may require a higher spatial resolution, although without the need for a weekly coverage of the Earth.

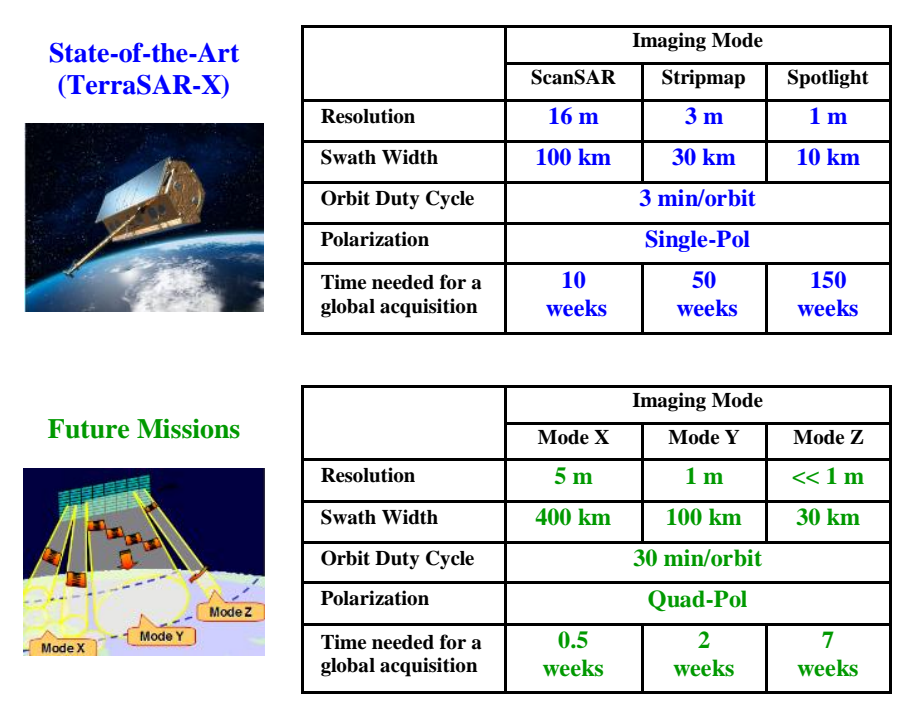

Figure 1: Requirements for spaceborne SAR imaging: Stateof-the-art (top) and future missions (bottom).

The latter requirements cannot be achieved by conventional SAR systems. A frequent coverage, in fact, requires that a wide swath is imaged. The swath width is though limited by the selected pulse repetition frequency (PRF): The swath can be widened, only if the PRF is reduced. However, a minimum $\mathrm{PRF}$ is required to guarantee a given azimuth resolution. Wide coverage can therefore be achieved only at the expense of a degraded azimuth resolution.

Among the key technologies, able to boost the performance of future SAR systems, there are digital beamforming (DBF) and multiple aperture signal recording. Several suggestions have been made to exploit these techniques to improve the imaging capabilities of SAR instruments. A prominent example is the high-resolution wide-swath (HRWS) SAR, currently under development at EADS Astrium with support from DLR [10]. This system uses DBF on receive to steer in real-time a narrow beam towards the direction of arrival of the radar echo from the ground (scan-on-receive). A large receiving antenna can hence be used to improve the sensitivity without narrowing the swath width. As the unambiguous swath width is limited by the antenna length, a long antenna is deployed to map a wide swath. Moreover, to improve the azimuth resolution, the receive antenna is divided into multiple sub-apertures, mutually displaced in the alongtrack direction and connected to individual receive channels. By this, multiple samples can be acquired for each transmitted pulse. The coherent combination of all signals in a dedicated 
multichannel processor enables the generation of a highresolution wide-swath SAR image [4].

The need for a very long antenna represents the main limitation of the mentioned system. In order to keep the antenna length down, several new instrument architectures and modes have been proposed in [6]. One example is the combination of displaced phase centers in azimuth with ScanSAR or TOPS mode (see top left of Fig. 2). As in classical ScanSAR, azimuth bursts are used to map several swaths. The associated resolution loss from sharing the synthetic aperture among different swaths is compensated by collecting the radar echoes with multiple displaced azimuth apertures. Besides multichannel ScanSAR, a concept based on multiple narrow elevation beams is also of interest, where each beam follows the echo of a different pulse transmitted by a wide beam illuminator. This enables an increase of the coverage area without the necessity to either lengthen the antenna or to employ burst modes. The top right of Fig. 2 provides an illustration, where three narrow receive beams follow the echoes from three simultaneously mapped image swaths that are illuminated by a broad transmit beam. A sufficiently high antenna is needed to separate the echoes from the different swaths by digital beamforming on receive. An interesting alternative to a planar antenna is a reflector, fed by a multichannel array, as illustrated on the lower left of Fig. 2. A parabolic reflector focuses an arriving plane wave on one or a small subset of feed elements. As the swath echoes arrive as plane waves from increasing look angles, one needs hence to only read out one feed element after the other to steer a high gain beam in concert with the arriving echoes. A drawback of the multi-beam mode is the presence of blind ranges across the swath, due to the fact that the radar cannot transmit and receive at the same time.

The Staggered-SAR concept overcomes this drawback by continuously varying the PRF along the track, so shifting the blind ranges across the swath (lower right of Fig. 2). HRWS imaging is thus achieved without the need for a long antenna with multiple apertures. This concept has been already analysed in [3], showing that a $350 \mathrm{~km}$ wide swath can be imaged with high resolution, although the proposed PRF variation schemes lead to a degradation of the azimuth impulse response, which in some cases is even dependent on the azimuth position of the target. In this paper, a new PRF variation scheme is proposed, which overcomes these drawbacks. Moreover, the proposed new scheme is able to ensure an overlap between the transmitted pulses and the echoes coming back from nadir, thus avoiding the image quality degradation associated with the incomplete suppression of the latters. Systems with continuously-varied PRF require that a uniformly sampled signal is recovered from a non-uniformly sampled one. This is usually done by using interpolation. In this paper multi-channel reconstruction is exploited as well. The performances are finally evaluated, comparing different processing approaches. Finally, the effects associated to the use of a continuously varied PRI are artificially introduced into real TerraSAR-X data acquired over Borneo, Indonesia, showing the resulting image degradation.

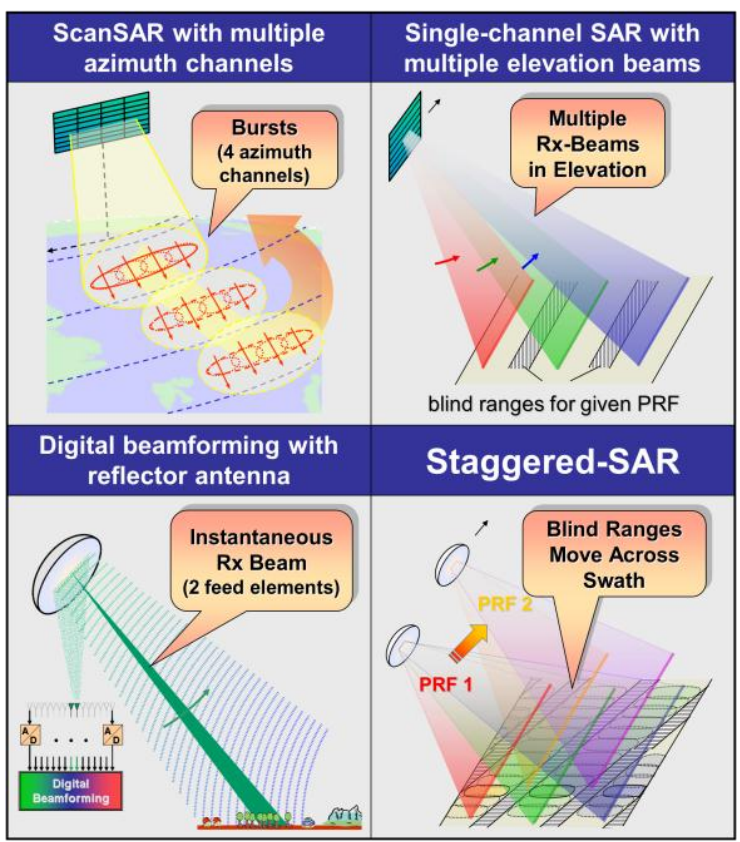

Figure 2: Advanced concepts for high-resolution wide-swath (HRWS) imaging.

\section{Continuous PRF Variation in SAR}

Conventional SAR systems employ a constant PRF. Once the sensor height $h_{s}$ and the uncompressed transmitted pulse duration $\tau$ are fixed, a timing diagram is obtained, like the one displayed in Fig. 3 for $h_{s}=760 \mathrm{~km}$ and $\tau=20 \mu \mathrm{s}$. This diagram shows, for each PRF, the available swaths on ground, which extend between two adjacent black strips. The red and green arrows in Fig. 3 delimit two available swaths for $P R F=$ $1600 \mathrm{~Hz}$ and $P R F=2500 \mathrm{~Hz}$, respectively. It can be noticed that the available swaths become narrower, as the PRF increases. Black strips correspond to blind ranges. The time it takes the transmitted pulse to reach those ranges and come back to the radar, in fact, is a integer multiple of the time interval between consecutive transmissions: The radar echoes coming back from those ranges, therefore, cannot be received, as the radar is transmitting ("blockage").

If the PRF is kept constant, there will therefore be some blind ranges, for which none of the transmitted pulses is received, while for all other ranges all the transmitted pulses will be received. In contrast, if the PRF is continuously varied, the ranges, from which the echoes will not be received, will be in general different for each transmitted pulse. From a different perspective, for each range, only some of the transmitted pulses will not be received - we will say they will be "blocked". In order to simplify the analysis of the system, it is convenient to consider a periodicity in the PRF variation, so that a sequence of $M$ intervals between transmissions or pulse repetition intervals (PRI) is employed. If these intervals are properly selected, quite a small percentage of transmitted pulses will be "blocked" for each range over a desired swath much larger than those achievable by a constant PRF system. 


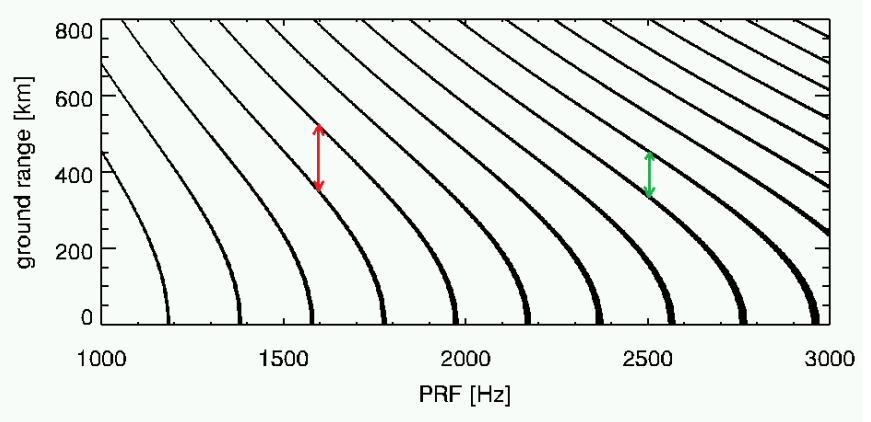

Figure 3: Timing diagram for a SAR system. Black stripes represent blind ranges due to "blockage".

An imaging system with continuously varied PRF has already been analysed in [3]. In particular, a slow linear PRI variation is considered, together with long sequences, with $M$ ranging from 580 up to 2900 pulses (corresponding to the full synthetic aperture). It is shown that this technique enables the imaging of a wide swath of $350 \mathrm{~km}$ with high resolution, although the resulting azimuth impulse response is characterised by the presence of several paired echoes in the vicinity of the main lobe and even varies with the azimuth position of the target, for $M=2900$.

The average percentage of blocked pulses is lower limited by twice the mean duty cycle, which is the ratio of the pulse length to the mean PRI [3]. If such percentage is approximately the same for all PRI variation schemes, the spatial distribution of the blocked pulses, i.e. the frequency and the size of the gaps, can be very different: Long sequences with slow-varying PRI lead to sporadic and large gaps, while short sequences with fast-varying PRI lead to frequent short gaps. The gaps in the raw data are responsible for the paired echoes in the azimuth impulse response, obtained after focusing the raw data. Moreover, the distance of these paired echoes from the main lobe is inversely proportional to the length of the sequence. This is the reason why several paired echoes appear in the vicinity of the main lobe, if a long sequences with slow PRI variation is used.

In contrast, if short sequences with fast PRI variation are employed, the paired echoes can be moved away from the main lobe. As the amplitudes of the paired echoes is also dependent on the azimuth antenna pattern, this leads to better performance, as it will be shown in the following sections. In order to avoid large gaps, in [6] a sequence of $M=40$ PRIs has been proposed, where two consecutive pulses are never blocked.

In this paper, we consider the design of very short sequences, following the criterion for which no more than one pulse of the sequence is blocked for ranges included in the desired swath. The idea underlying this criterion is to minimise the length of the sequence, in order to move the paired echoes as far as possible away from the main lobe. If a linear PRI variation is considered, sequences of $M=2, M=3, M=5$, or $M=7$ PRIs can be obtained, which obey to the above mentioned criterion. The expressions of the $M$ PRIs, $P R I_{k}, k=$ $0 . . M-1$, are obtained by imposing that "anyhow two distinct sets of $p$ and $q(p, q<M)$ consecutive PRIs of the sequence are taken, the difference of the sum of the PRIs of the two sets is always greater than $2 \tau$ (or at least equal to $2 \tau$ )", where the word "consecutive" considers the cyclicality of the sequence $\left(P R I_{M-1}\right.$ and $P R I_{0}$ are two consecutive PRIs). It holds:

$$
\begin{aligned}
& P R I_{0}=\frac{\left(M^{2}+2 M+1\right)}{4} \Delta \\
& P R I_{k}=P R I_{0}-k \Delta, \quad k=1 . . M-1 \\
& \Delta \geq 2 \tau
\end{aligned}
$$

where $\Delta$ is an arbitrary time interval, greater or equal than $2 \tau$. Fig. 4 shows the sequence of transmitted pulses, having selected the PRIs according to (1), for $M=5, \Delta=72.4 \mu \mathrm{s}$, and $\tau=36 \mu \mathrm{s}$. As the sequence of PRIs repeats periodically, one period is displayed.

In Fig. 5 the received (blue) and blocked (red) pulses are superimposed to the transmitted ones (black) for a ground range of $520 \mathrm{~km}$ and a sensor height $h_{s}=760 \mathrm{~km}$. The numbers close to the pulses indicate the number of pulse within the sequence ( 1 to $M$ ). It can be noticed that the second transmitted pulse cannot be received (or, better, can be only partially received), as the system is transmitting the fourth pulse of the sequence. Partially received pulses are ignored (discarded) in our analysis, even though additional information might be gathered from them as well.

As mentioned, the plot of Fig. 5 refers to a specific ground range. For different ranges the relative shift between transmitted and received pulses changes and the blocked pulse changes as well. The diagram of Fig. 6 shows which transmitted pulse of the sequence is blocked for each ground range. As apparent, no more than one pulse is blocked over a ground range, ranging from $100 \mathrm{~km}$ to more than $800 \mathrm{~km}$.

Furthermore, all echoes coming back from nadir are blocked. This is obtained by imposing that the time it takes the pulse to reach nadir and come back to the radar is an integer multiple of the period of the sequence $T_{\text {seq }}$

$$
\frac{2 h_{s}}{c_{0}}=k T_{s e q}
$$

where $c_{0}$ is the speed of light in free space and $T_{\text {seq }}$ is the period of the sequence, which can be expressed as

$T_{\text {seq }}=\sum_{i=0}^{M-1} P R I_{i}=\frac{M\left(M^{2}+3\right)}{4} \Delta$

In this example, $k$ is equal to 2 .

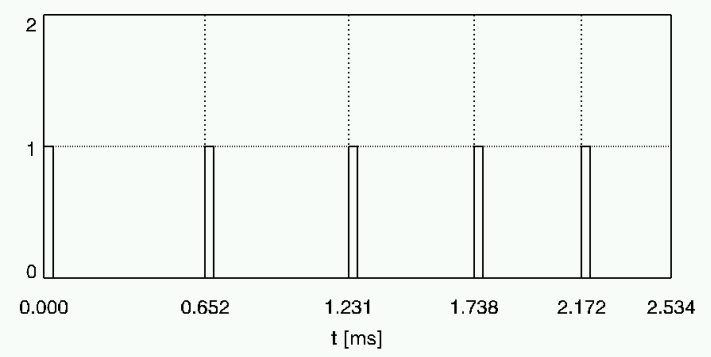

Figure 4: Sequence of $M=5$ transmitted pulses with linear PRI variation. 
If the PRIs are defined as in (1), no more than one pulse out of $M$ will be blocked. This also guarantees a rather small maximum separation between received ("non-blocked") samples. Fig. 7 shows the maximum, mean, and minimum pulse separation between the received pulses. Depending on the required azimuth resolution, a minimum azimuth bandwidth has to be processed. If the required azimuth bandwidth is smaller than the reciprocal of the processed Doppler bandwidth, then the maximum pulse separation is even smaller than the sampling period required by the Nyquist criterion. In contrast, the maximum pulse separation is by far longer, if the long sequences proposed in [3] are used (up to $32 \mathrm{~ms}$ for $M=580$ and up to $160 \mathrm{~ms}$ for $M=2900$ ).

The minimum pulse separation has to be kept into account as well. In order to suppress range ambiguities, in fact, a higher antenna is required, if the minimum pulse separation is smaller [6].

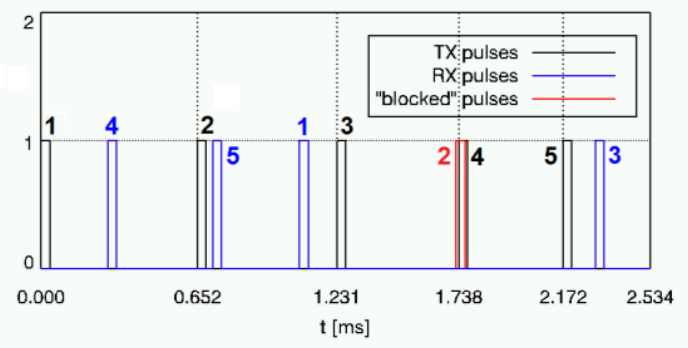

Figure 5: Transmitted, received, and blocked samples for a ground range of $520 \mathrm{~km}$.

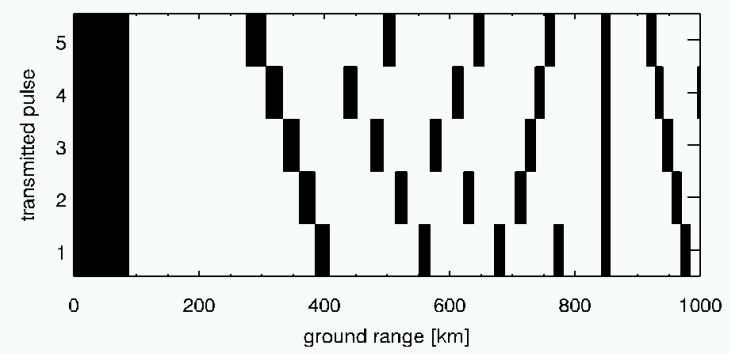

Figure 6: "Blocked" transmitted pulses (black) for each range.

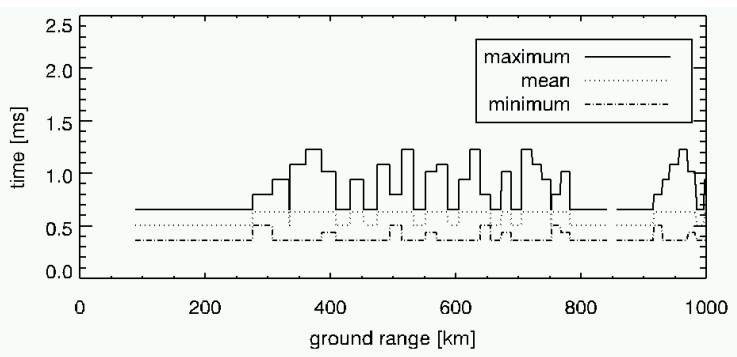

Figure 7: Maximum, mean and minimum pulse separation.

\section{Signal Processing}

As a consequence of the use of a continuously varied PRI and due to the presence of "blocked" pulses, a non-uniformly sampled gapped raw azimuth signal is available.
Before performing azimuth focusing, a uniformly sampled raw azimuth signal has to be recovered from the nonuniformly sampled gapped one. This can be done using interpolation techniques, as proposed in [3].

As an alternative, a promising approach is based on the generalised sampling expansion which recovers the uniformly sampled signal by means of multi-channel reconstruction [2]. If $N$ is the number of received ("non-blocked") pulses within the sequence $(N \leq M)$, according to the generalised sampling expansion [9], the available non-uniformly sampled raw azimuth signal allows the perfect recovery of a signal bandlimited to $\left[-1 /\left(2 \cdot N \cdot T_{\text {seq }}\right), 1 /\left(2 \cdot N \cdot T_{\text {seq }}\right)\right]$. In particular, an equivalent uniformly-sampled signal can be obtained by means of $N$ post-filters [1]. As the received azimuth signal is not band-limited, a reconstruction error will be present, as the energy outside the above mentioned frequency band will disturb the reconstruction of the signal. An expression for the resulting spectral error is provided in [2]. Some investigations concerning the use of the multi-channel reconstruction in systems with continuously varied PRF have been reported in [11].

In this paper, we consider a design example, using the sequence of 5 PRIs of Fig. 4 together with a set of system parameters typical of the Tandem-L mission [7]. Fig. 8 shows the original and recovered received raw azimuth signal from a point-target, using both interpolation and multi-channel reconstruction. The example refers to a ground range of 520 $\mathrm{km}$ and represents the worst case: The second of the five transmitted pulses is blocked (cf. Fig. 5), leading to the maximum pulse separation (cf. Fig. 7). It can be noticed that multi-channel reconstruction provides an almost perfect recovery of the high-amplitude (low-frequency) components. In particular, the energy of the reconstruction error is equal to $9.6 \%$ and $2.8 \%$ of the energy of the original signal, for interpolation and multi-channel reconstruction, respectively.
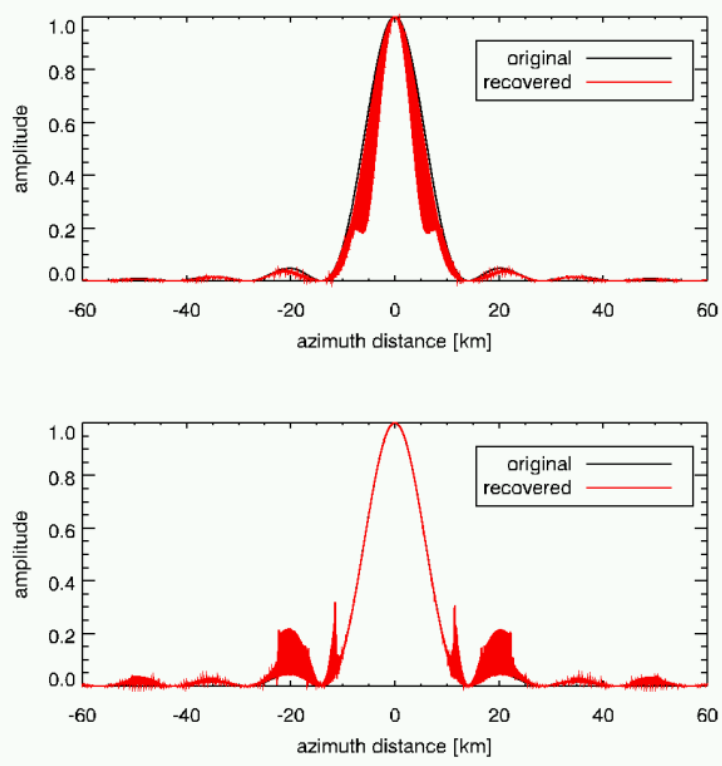

Figure 8: Original and recovered raw azimuth signals, using interpolation (top) and multi-channel reconstruction (bottom). 
The recovered uniformly-sampled raw azimuth signal is then focused using a conventional SAR processor. The effects of range cell migration (RCM) and their implication on the $2 \mathrm{D}$ signal reconstruction are also a concern. The relative range offset, however, is likely to be negligible. If not, RCM can be corrected as suggested in [4].

\section{Performance Analysis}

The non-perfect recovery of the raw azimuth signal determines a degradation of the azimuth impulse response: This could include in general an enlargement of the main lobe (worse azimuth resolution), higher side-lobes, as well as the presence of additional paired echoes.

Fig. 9 shows the azimuth impulse responses, obtained after focusing the recovered raw signals of Fig. 8, using interpolation and multi-channel reconstruction. The impulse responses are superimposed to the reference uniform sampling impulse response. An azimuth Doppler bandwidth of $900 \mathrm{~Hz}$ has been processed, while no azimuth windows (e.g. Hamming) have been applied. It can be noticed that using the very short sequences proposed in this paper there are no paired echoes in the vicinity of the main lobe.

Fig. 10 shows the same impulse responses of Fig. 9, but the $\mathrm{x}-$ axis (azimuth distance) is enlarged to $120 \mathrm{~km}$, so that azimuth ambiguities are also visible. It can be noticed that some paired echoes appear in the impulse response in addition to azimuth ambiguities. The distances of the echoes from the main lobe are integer multiples of a quarter of the first azimuth ambiguity's distance from the main lobe itself. This is related to the fact that four different pulse separations are present in the azimuth raw data (one pulse out of five is blocked). The echoes' amplitudes are smaller, if multi-channel reconstruction is used. We emphasise once more that the plots refer to a worst case (ground range equal to $520 \mathrm{~km}$ ): The echoes' amplitude are much smaller for other ranges.

A quantitative assessment of the impulse response degradation is provided by the integrated side-lobe ratio (ISLR), defined as the ratio of the energy of all side-lobes to the main lobe energy. Fig. 11 shows the ISLR as a function of ground range, using both interpolation and multi-channel reconstruction. Compared to the uniform sampling reference case (ISLR $=-14.5 \mathrm{~dB}$ ), the ISLR loss is never larger than 3 $\mathrm{dB}$ and $1.5 \mathrm{~dB}$, for interpolation and multi-channel reconstruction, respectively. For the majority of ranges, however, the ISLR is the same as in the uniform sampling case. Using the long sequences proposed in [3], the ISLR loss reaches $6 \mathrm{~dB}$, for $\tau=20 \mu \mathrm{s}$, and is likely to be much larger for $\tau=35 \mu \mathrm{s}$.

In addition to the ISLR, the noise scaling factor [2], associated with the recovery of the uniformly-sampled raw azimuth signal, has to be considered. Fig. 12 shows the noise scaling factor for the considered design example. When using multi-channel reconstruction, the noise amplification could become critical, reaching $6 \mathrm{~dB}$.

A joint optimisation of the sequence, system, and processing parameters is expected to lead to improved performance.
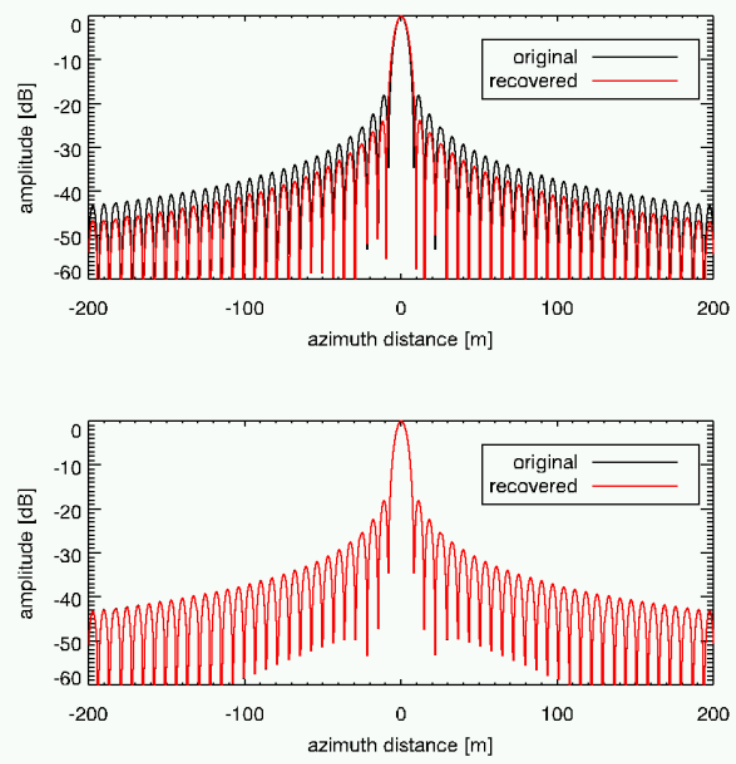

Figure 9: Azimuth impulse responses, using interpolation (top) and multi-channel reconstruction (bottom). The $\mathrm{x}$-axis (azimuth distance) extends from $-200 \mathrm{~m}$ to $200 \mathrm{~m}$.
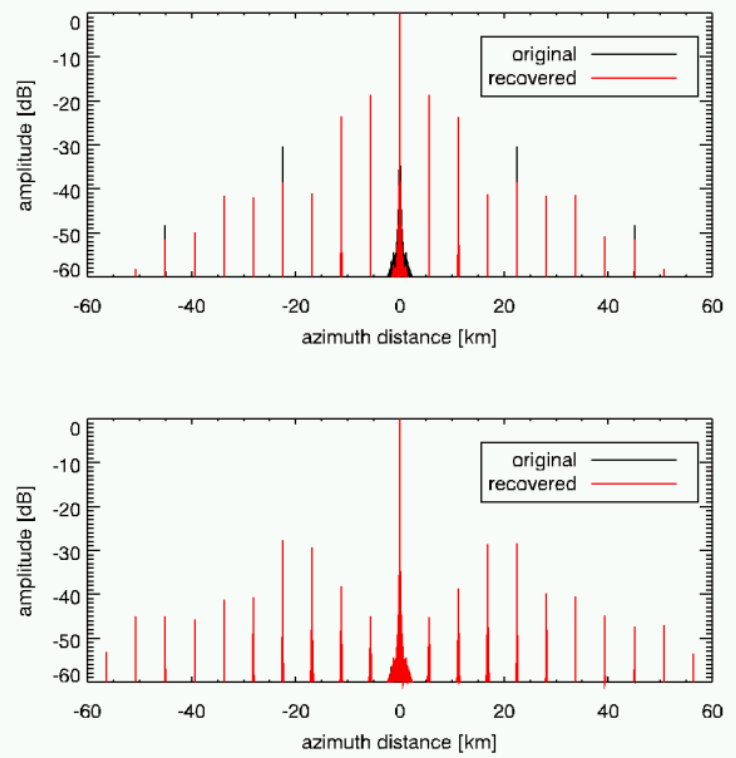

Figure 10: Azimuth impulse response, using interpolation (top) and multi-channel reconstruction (bottom). The $\mathrm{x}$-axis (azimuth distance) extends from $-60 \mathrm{~km}$ to $60 \mathrm{~km}$.

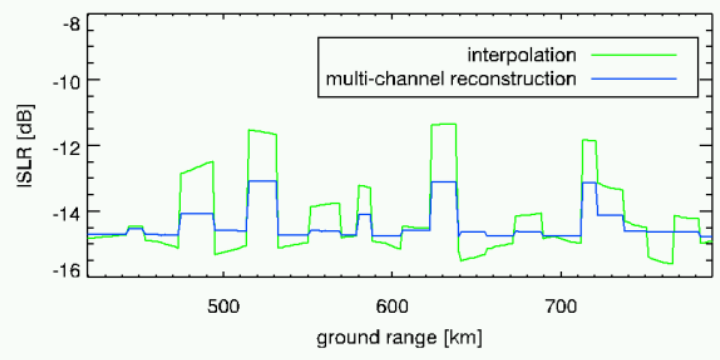

Figure 11: ISLR $(\mathrm{dB})$ for interpolation and multi-channel reconstruction. 


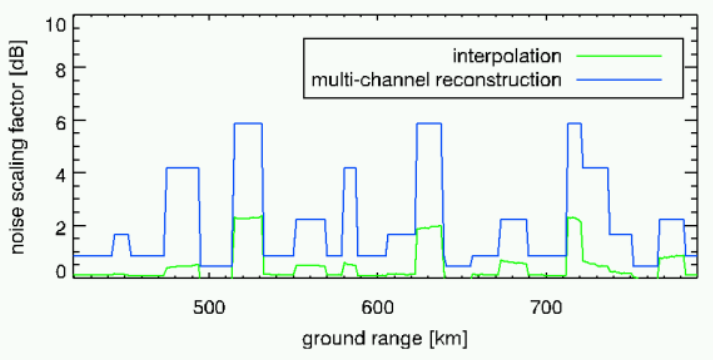

Figure 12: Noise scaling factor $(\mathrm{dB})$ for interpolation and multi-channel reconstruction.

To give and idea of how paired echoes and noise amplification impact the SAR image quality in the worst case, we have artificially introduced the paired echoes and the increased noise associated to interpolation and multi-channel reconstruction in a TerraSAR-X data set, acquired over Borneo, Indonesia (Fig. 13). While for interpolation the effects of the paired echoes are visible (see replicated pointlike target on the left-hand side of the image), they are hardly noticeable for multi-channel reconstruction, where the image contrast is on the other hand more impacted by the increased noise.

The two processing approaches should not be seen as exclusive. In the critical worst case, if both images are available, depending on the presence of strong targets at specific distances, one could choose between the two images or a combination of them, even on a pixel-by-pixel basis.
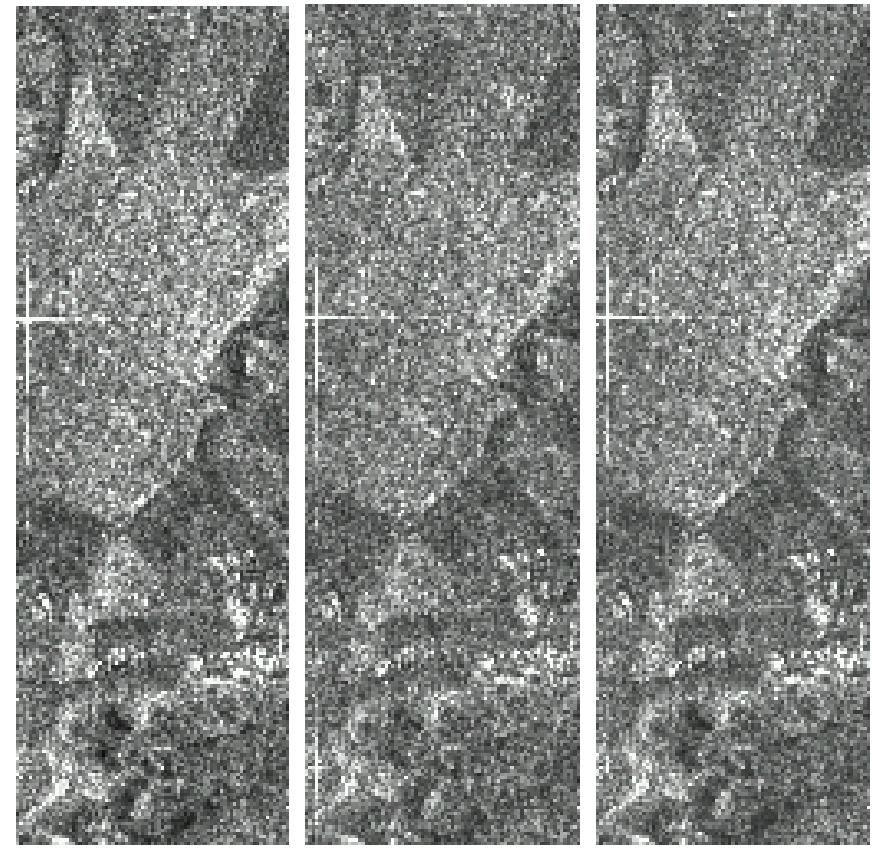

Figure 13: Artificial introduction of paired echoes and noise amplification in a TerraSAR-X image acquired over Borneo, Indonesia. Original (left) and degraded images, using interpolation (centre) and multi-channel reconstruction (right).

\section{Conclusions}

Staggered SAR allows HRWS imaging without the need for a long antenna with multiple apertures. The system gets rid of blind ranges by continuously varying the PRF along the track. This paper proposes the use of very short sequences of PRIs and considers multi-channel reconstruction, as a possibility to recover a uniformly sampled raw azimuth signal in addition to interpolation. The analysis shows that, if very short sequences are used, the paired echoes in the impulse response can be moved away from the main lobe and nadir echoes can be suppressed, thus leading to improved performance.

Even though some aspects are not fully understood, the first results suggest that the Staggered-SAR concept will play an important role in the design of future spaceborne SAR systems with high-resolution wide-swath capability.

\section{References}

[1] J. L. Brown. "Multi-Channel Sampling of Low-Pass Signals", IEEE Trans. Circ. Sys., 28 (2), pp. 101-106, (1981).

[2] N. Gebert, G. Krieger, A. Moreira. "Digital Beamforming on Receive: Techniques and Optimization Strategies for High-Resolution Wide-Swath SAR Imaging", IEEE Trans. Aero. El. Sys., 45 (2), pp. 564592, (2009).

[3] N. Gebert, G. Krieger. "Ultra-Wide Swath SAR Imaging with continuous PRF Variation", Proc. EUSAR, Aachen, Germany, pp. 966-969, (2010)

[4] G. Krieger, N. Gebert, A. Moreira. "Unambiguous SAR signal reconstruction from nonuniform displaced phase center sampling", IEEE Geo. Rem. Sens. Lett., 1, pp. 260-264, (2004).

[5] G. Krieger et al. "TanDEM-X: A Satellite Formation for High-Resolution SAR Interferometry”, IEEE Trans. Geo. Rem. Sens., 45 (11), pp. 3317-3341, (2007).

[6] G. Krieger et al. "Advanced Concepts for Ultra-WideSwath SAR Imaging", Proc. EUSAR, Friedrichshafen, Germany, pp. 31-34, (2008).

[7] G. Krieger et al. "The Tandem-L Mission Proposal: Monitoring Earth's Dynamics with High Resolution SAR Interferometry," Proc. IEEE Radar Conference (RadarCon), Pasadena, CA, USA, (2009).

[8] G. Krieger et al. "Single-Pass Synthetic Aperture Radar (SAR) Missions", Proc. IEEE - Special Issue on Satellite Remote Sensing Missions for Monitoring Water, Carbon and Global Climate Change, 98 (5), pp. 816-843, (2010).

[9] A. Papoulis. "Generalised Sampling Expansion", IEEE Trans. Circ. Sys., 24 (11), pp. 652-654, (1977).

[10] M. Suess, B. Grafmueller, R. Zahn. "A novel high resolution, wide swath SAR system", Proc. IGARSS, Sydney, Australia, (2001)

[11] M. Villano, G. Krieger, A. Moreira. "Staggered-SAR: A New Concept for High-Resolution Wide-Swath Imaging", Proc. IEEE GOLD, Rome, Italy, (2012) 